\section{Perfil do consumo de bebidas alcoólicas e fatores associados em um município do Nordeste do Brasil}

\author{
Alcohol consumption and associated factors \\ in a city in Northeast Brazil
}

\author{
1 Departamento de Saúde, \\ Universidade Estadual do \\ Sudoeste da Bahia, Jequié, \\ Brasil. \\ 2 Instituto Multidisciplinar \\ em Saúde, Universidade \\ Federal da Bahia, Vitória da \\ Conquista, Brasil. \\ Correspondência \\ L. N. Ferreira \\ Departamento de Saúde, \\ Universidade Estadual do \\ Sudoeste da Bahia. \\ Rua Moisés Caroso 469, \\ Jequié, BA 45205-180, Brasil. \\ lnery@uesb.edu.br
}

\section{Abstract}

This cross-sectional study focused on alcohol consumption according to gender, age, income, and schooling in Jequié, Bahia State, Brazil. The random sample consisted of 270 individuals over 14 years of age living in the urban area of Jequié from January to March 2010. Alcohol consumption was assessed using the Alcohol Use Disorders Identification Test (AUDIT). Of the total sample, $21.8 \%$ consumed four or more drinks on a typical day, $14.1 \%$ admitted having caused any harm after drinking, and $26.3 \%$ reported that someone had already suggested they should stop drinking. Forty percent of men had more than four drinks on a typical day, and $50.5 \%$ of young people drank frequently. Distribution of the variables was consistent with higher frequency and greater amounts of alcohol among men and young people. There was a large proportion of heavy episodic alcohol consumption. The study identified vulnerable groups that are still not addicted to alcohol, and that should be targeted for public health promotion and prevention policies.

Alcohol-Related Disorders; Alcohol Drinking; Cross-Sectional Studies
Luciano Nery Ferreira 1 Zenilda Nogueira Sales 1 Cezar Augusto Casotti 1 José Patrício Bispo Júnior 2 Antônio Carlos Ricardo Braga Júnior ${ }^{2}$

\section{Introdução}

O consumo de bebidas alcoólicas é um hábito pré-histórico. Há evidências arqueológicas da sua utilização em celebrações, ritos ou simples aglomerações de indivíduos. Com o processo civilizatório, o consumo de bebidas alcoólicas foi incorporado aos padrões sociais aceitáveis. No entanto, a associação do consumo de álcool com a dependência, comportamentos antissociais e violência deixou ao longo da história a percepção de que há um limite tênue entre o consumo aceitável e os seus efeitos psicotrópicos.

No Brasil o consumo de bebidas alcoólicas antecede a chegada dos portugueses. O cauim bebida resultante da fermentação do milho e da mandioca - já era produzido e consumido pelos índios. Com a colonização portuguesa e a disponibilidade da cana-de-açúcar, popularizou-se a cachaça, que é uma bebida destilada com teor alcoólico consideravelmente alto 1 .

Não obstante a longa convivência da humanidade com o consumo de bebidas alcoólicas, pode-se considerar que se trata de um grave problema de saúde pública 2 relacionado a diversos agravos à saúde 3 . O consumo de bebidas alcoólicas influencia diretamente o perfil de morbimortalidade, tanto em sua relação com a violência 4,5,6 quanto pelo aumento do risco de doenças crônicas como hipertensão, cirrose, neoplasias. Apesar de serem relatados os benefícios do consumo moderado de bebidas alcoó- 
licas como fator protetor para algumas doenças cardiovasculares, os efeitos deletérios do consumo de álcool predominam sobre as potenciais vantagens 7 .

Acerca dos problemas de saúde relacionados ao consumo de bebidas alcoólicas, destacam-se a intoxicação alcoólica aguda, o uso nocivo para a saúde, também chamado de uso abusivo e o alcoolismo crônico ou síndrome de dependência. A intoxicação alcoólica aguda é caracterizada por um estado agudo e transitório de perturbação da consciência e/ou do estado cognitivo secundário ao consumo de bebida alcoólica. O uso abusivo compreende um modo de consumo caracterizado pelas complicações físicas e/ou psíquicas. Já o alcoolismo crônico, ou síndrome de dependência, é caracterizado como um conjunto de fenômenos comportamentais, cognitivos e fisiológicos que se desenvolvem após repetido consumo de bebida alcoólica, tipicamente associado ao desejo poderoso de consumi-la, à dificuldade de controlar o consumo, à utilização persistente, a uma maior prioridade dada ao seu uso em detrimento de outras atividades e obrigações, a um aumento da tolerância de doses e por vezes, a um estado de abstinência física 8 .

Estima-se que $20 \%$ a $40 \%$ dos pacientes internados em hospitais gerais apresentam problemas relacionados ao uso do álcool e que 5\% das mortes de pessoas entre 15 e 29 anos em todo o mundo estejam relacionadas ao uso excessivo de álcool 9 .

A Organização Mundial da Saúde (OMS) 10 estima que 2 bilhões de pessoas no mundo consomem álcool e 76,3 milhões possuem diagnóstico de consumo abusivo. O consumo de álcool é responsável por 1,8 milhões de mortes por ano, 3,2\% da mortalidade total, e $4 \%$ dos anos potencias de vida perdidos. Um terço dessas mortes é atribuído aos acidentes e causas não intencionais.

Recentemente, o II Levantamento Domiciliar sobre o Uso de Drogas Psicotrópicas no Brasil 11 apurou uma prevalência de $74,6 \%$ de uso de álcool na vida e 12,3\% de dependência de álcool no Brasil. Neste cenário, a Região Nordeste desponta com a maior prevalência de dependência, com $13,8 \% 11$.

Diversos estudos apontam para uma maior prevalência da dependência de álcool entre homens do que entre mulheres 11, embora esta tendência esteja diminuindo com a emancipação feminina 12. Com relação à idade, embora as maiores prevalências sejam encontradas entre adultos jovens, chama atenção a grande quantidade de adolescentes que fizeram uso na vida de álcool e dos que já são dependentes 11. O uso precoce de álcool demanda preocupação na medida em que quanto mais cedo o jovem começa a beber, mais danoso será o seu padrão de consumo de álcool no futuro ${ }^{13}$.

Fatores locais e regionais ligados às variáveis sociodemográficas exercem influência diferenciada nos padrões de consumo de bebidas alcoólicas e na dependência do álcool 14. Grandes centros urbanos brasileiros já foram pesquisados a este respeito 11,14,15,16, no entanto, ainda há lacunas quanto ao conhecimento epidemiológico deste fator em municípios de pequeno e médio porte. Neste sentido, este estudo teve por objetivo verificar o perfil do consumo de bebidas alcoólicas de acordo com sexo, idade, renda e escolaridade na população urbana de Jequié, Bahia.

\section{Metodologia}

Estudo epidemiológico transversal tipo inquérito domiciliar de base populacional, com indivíduos com idade maior ou igual a 14 anos residentes na zona urbana do Município de Jequié. Adotou-se a contagem populacional de 2007 do Instituto Brasileiro de Geografia e Estatística (IBGE. http:// www.ibge.gov.br), 145.964 habitantes. Descontados os habitantes da zona rural e os menores de 14 anos vivendo na zona urbana, o universo do estudo foi de 99.941 indivíduos. O cálculo amostral, realizado com o programa Epi Info versão 3.5.1 (Centres for Disease Control and Prevention, Atlanta, Estados Unidos), levou em consideração dados de prevalência da dependência de álcool para a Região Nordeste do Brasil no ano de $200511,13,8 \%$, margem de erro de $5 \%$, o que apontou para uma amostra inicial de 129 indivíduos, adotando o nível de $90 \%$ de confiança. A partir daí foram feitos ajustes para efeito desenho, usando o fator de correção 2 e ajustes para perdas amostrais, prevendo uma taxa de não resposta máxima de $10 \%$ para uma amostra sem reposição, o que resultou em uma amostra de 284 indivíduos.

A amostra foi estratificada por conglomerados, em três estágios, respectivamente: setores censitários, domicílio e indivíduo. Para pesquisar este contingente, 15 setores censitários urbanos de um total de 148 foram sorteados de forma aleatória sistemática. Em cada setor, vinte domicílios foram sorteados e visitados, no período de janeiro a março de 2010, por uma equipe de seis pesquisadores treinados. Considerando uma média de 250 domicílios por setor, adotou-se um intervalo sistemático de 12 domicílios. Em cada setor as quadras foram enumeradas e dispostas sequencialmente. O setor foi percorrido em suas quadras no sentido horário, considerados os domicílios do lado direito do logradouro pertencente à quadra. As quadras foram sucessivamente 
percorridas até atingir os vinte domicílios sorteados em cada setor censitário.

Foi considerado domicílio o local estruturalmente separado e independente que se destina a servir de habitação a uma ou mais pessoas, ou que estivesse sendo utilizado com esta finalidade. Foram excluídos estabelecimentos onde existisse relação de convivência de longa permanência de caráter institucional como abrigos, asilos, presídios, casas de acolhimento noturno, quartéis, orfanatos, conventos, hospitais ou alojamentos de trabalhadores.

Em cada domicílio, o sorteio do indivíduo a ser entrevistado foi feito por meio da contagem sequencial contínua dos residentes no domicílio com idade maior ou igual a 14 anos, dispostos em ordem crescente de idade, até que se atingisse um número aleatório, que foi previamente definido e impresso na capa do formulário de entrevista.

Funcionários como cuidadores de idosos, babás e empregadas domésticas só foram incluídos no sorteio se efetivamente residissem naquele domicílio. Trabalhadores que retornavam para o seu domicílio diariamente não entraram no sorteio do local de trabalho. Por outro lado, nos domicílios em que o indivíduo sorteado não se encontrava no momento da visita do entrevistador, foi agendada uma visita em horário cômodo para o indivíduo a ser entrevistado. Foram admitidas quatro tentativas de visita para entrevistar o indivíduo.

O instrumento utilizado compunha-se de variáveis sociodemográficas e de questões relacionadas ao consumo do álcool. Para tanto, foram utilizadas as versões brasileiras do Alcohol Use Disorders Identification Test (AUDIT) 17 e do Cutdown, Annoyed, Guilty e Eye-opener (CAGE) 18.

O AUDIT é um questionário relacionado ao padrão de consumo de bebidas alcoólicas, traduzido e validado para o Brasil, com capacidade de verificar os domínios de uso arriscado do álcool, sintomas de dependência e uso nocivo. Apresenta alta sensibilidade e especificidade para utilização na população brasileira 19. Adotou-se como ponto de corte o escore $\geq 8$ obtido no AUDIT para definir uso arriscado ou prejudicial de álcool 17 .

O questionário CAGE, traduzido e validado para o Brasil 18 , é utilizado para o rastreamento epidemiológico de dependência do álcool, adotando-se duas ou mais respostas positivas como ponto de corte.

A análise estatística utilizou o teste do $\chi^{2}$ de Pearson para verificar a associação entre os itens que compõem o AUDIT e as variáveis gênero, faixa etária, escolaridade, renda familiar e a classificação segundo escore obtido no AUDIT. Adotou-se intervalo de 95\% de confiança (IC95\%). Todos os cálculos foram realizados pelo programa R versão 2.9.0 (The R Foundation for Statistical Computing, Viena, Áustria; http:// www.r-project.org).

A pesquisa foi analisada e autorizada pelo Comitê de Ética em Pesquisa da Universidade Estadual do Sudoeste da Bahia (protocolo 044/2009). Foram observados todos os dispostos acerca da ética em pesquisa com seres humanos, conforme preconizado pela Resolução $n^{o}$. 196/96 do Conselho Nacional de Saúde.

\section{Resultados}

Foram pesquisados 270 indivíduos, dado que a taxa de não resposta real foi de $5 \%$. A média de idade foi de 39 anos, desvio-padrão 17,4. A amostra obtida foi composta de $60,7 \%$ do sexo feminino e $39,3 \%$ masculino.

Na Tabela 1, são descritos todos os itens do perfil de consumo de bebidas alcoólicas do AUDIT. Para o item frequência de consumo, observa-se que $3,7 \%$ das pessoas consomem bebidas alcoólicas duas ou mais vezes por semana. No que diz respeito à quantidade de doses que se bebe num dia típico, $21,8 \%$ das pessoas relataram consumir quatro ou mais doses. A respeito da frequência com que se consomem seis ou mais doses em uma ocasião, 13,3\% das pessoas referiram que o fazem pelo menos uma vez ao mês. Quando perguntados sobre terem sentido culpa ou remorso após ter bebido, $12,9 \%$ referiram que isso aconteceu pelo menos uma vez no último ano. A respeito da ocorrência, no último ano, de episódios de amnésia em seguida a uma bebedice, $7,8 \%$ das pessoas confirmaram a ocorrência de pelo menos um episódio. Ao serem questionados sobre já terem causado algum prejuízo a si mesmo ou a outrem após ter bebido, $14,1 \%$ das pessoas admitiram que sim. Sobre o aconselhamento de terceiros para que diminuíssem a bebida ou parassem de beber, $26,3 \%$ das pessoas relataram já terem sido sugeridas por algum parente ou profissional de saúde.

Em todos os itens, as prevalências de respostas compatíveis com perfil de beber de risco foram mais elevadas no sexo masculino (Tabela 1). Foi encontrada significância estatística entre 0 sexo e oito dos dez itens do perfil de consumo estabelecidos pelo AUDIT. Apenas os itens necessidade de bebida pela manhã e deixar de fazer o esperado devido à bebida não se mostraram significativamente associados com sexo. No que se refere ao número de doses, observa-se que entre as mulheres, $90 \%$ delas não consomem mais do que três doses em dia típico, ao passo que, entre os homens $40 \%$ bebem mais que quatro doses no dia típico. Destaca-se ainda que $17 \%$ dos homens 
Tabela 1

Distribuição do perfil de consumo de bebidas alcoólicas (em \%) segundo o gênero. Município de Jequié, Bahia, Brasil, 2010.

\begin{tabular}{|c|c|c|c|c|}
\hline Variável & Homens $(n=106)$ & Mulheres $(n=164)$ & Valor de $p$ * & $\begin{array}{c}\text { Ambos ( } n=270) \\
\text { Prevalência (IC95\%) }\end{array}$ \\
\hline Frequência de consumo & & & 0,007 & \\
\hline Nunca & 44,3 & 64,6 & & $56,7(50,8-62,6)$ \\
\hline Mensalmente ou menos & 20,8 & 15,9 & & $17,8(13,2-22,4)$ \\
\hline $2-4$ vezes/mês & 30,2 & 16,5 & & $21,9(17,0-26,8)$ \\
\hline $2-3$ vezes/semana & 4,7 & 1,8 & & $3,0(1,0-5,0)$ \\
\hline 4 ou mais vezes/semana & - & 1,2 & & $0,7(0,0-1,7)$ \\
\hline Número de doses em dia típico & & & $<0,001$ & \\
\hline $0-1$ & 49,1 & 75,0 & & $64,8(59,1-70,5)$ \\
\hline $2-3$ & 10,4 & 15,2 & & $13,3(9,2-17,4)$ \\
\hline $4-5$ & 14,2 & 6,7 & & $9,6(6,1-13,1)$ \\
\hline $6-7$ & 9,4 & 1,2 & & $4,4(2,0-6,8)$ \\
\hline 8 ou mais & 17,0 & 1,8 & & $7,8(4,6-11,0)$ \\
\hline $\begin{array}{l}\text { Frequência de seis ou mais doses em uma } \\
\text { ocasião }\end{array}$ & & & $<0,001$ & \\
\hline Nunca & 56,6 & 84,1 & & $73,3(68,0-78,6)$ \\
\hline Menos que mensalmente & 19,8 & 9,1 & & $13,3(9,2-17,4)$ \\
\hline Pelo menos 1 vez ao mês & 23,6 & 6,7 & & $13,3(9,2-17,4)$ \\
\hline Frequência de beber sem conseguir parar & & & 0,024 & \\
\hline Nunca & 90,6 & 97,6 & & $94,8(92,2-97,4)$ \\
\hline Menos que mensalmente & 7,5 & 1,2 & & $3,7(1,4-6,0)$ \\
\hline Pelo menos 1 vez ao mês & 1,9 & 1,2 & & $1,5(0,1-2,9)$ \\
\hline Deixou de fazer o esperado devido à bebida & & & 0,238 & \\
\hline Nunca & 93,4 & 97,6 & & $95,9(93,5-98,3)$ \\
\hline Menos que mensalmente & 4,7 & 1,8 & & $3,0(1,0-5,0)$ \\
\hline Pelo menos 1 vez ao mês & 1,9 & 0,6 & & $1,1(0,0-2,3)$ \\
\hline Necessidade de bebida pela manhã & & & 0,482 & \\
\hline Nunca & 92,5 & 95,7 & & $94,4(91,7-97,1)$ \\
\hline Menos que mensalmente & 4,7 & 3,0 & & $3,7(1,4-6,0)$ \\
\hline Pelo menos 1 vez ao mês & 2,8 & 1,2 & & $1,9(0,3-3,5)$ \\
\hline Sentiu-se culpado depois de beber & & & 0,003 & \\
\hline Nunca & 78,3 & 92,7 & & $87,0(83,0-91,0)$ \\
\hline Menos que mensalmente & 17,0 & 5,5 & & $10,0(6,4-13,6)$ \\
\hline Pelo menos 1 vez ao mês & 4,7 & 1,8 & & $3,0(1,0-5,0)$ \\
\hline Incapaz de lembrar o que aconteceu devido à & & & $<0,001$ & \\
\hline bebida & & & & \\
\hline Nunca & 84,0 & 97,6 & & $92,2(89,0-95,4)$ \\
\hline Menos que mensalmente & 13,2 & 1,2 & & $5,9(3,1-8,7)$ \\
\hline Pelo menos 1 vez ao mês & 2,8 & 1,2 & & $1,9(0,3-3,5)$ \\
\hline Causou ferimentos ou prejuízos após ter bebido & & & $<0,001$ & \\
\hline Não & 73,6 & 93,9 & & $85,9(81,7-90,1)$ \\
\hline Sim, mas não nos últimos 12 meses & 18,9 & 4,3 & & $10,0(6,4-13,6)$ \\
\hline Sim, nos últimos 12 meses & 7,5 & 1,8 & & $4,1(1,7-6,5)$ \\
\hline Alguém sugeriu que parasse de beber & & & $<0,001$ & \\
\hline Não & 53,8 & 86,6 & & $73,7(68,4-79,0)$ \\
\hline Sim, mas não nos últimos 12 meses & 25,5 & 6,1 & & $13,7(9,6-17,8)$ \\
\hline Sim, nos últimos 12 meses & 20,8 & 7,3 & & $12,6(8,6-16,6)$ \\
\hline
\end{tabular}

IC95\%: intervalo de 95\% de confiança.

* Teste $\chi^{2}$ 
bebem mais que oito doses em dia típico, proporção consideravelmente maior à observada entre as mulheres, $1,8 \%$. Entre os homens, também se observou maior proporção no item causou ferimentos ou prejuízo após ter bebido. Enquanto $6,1 \%$ das mulheres relataram prejuízos a si ou a terceiros por causa da bebida, entre os homens esse percentual eleva-se para $26,4 \%$. No que se refere à sugestão de outrem sobre parar de beber, entre os homens, 46,2\% já foram aconselhados a abandonar o hábito da bebida. Entre as mulheres, o percentual é de apenas 13,4\%.

A análise da distribuição do perfil de consumo de bebidas alcoólicas por faixas etárias (Tabela 2) revelou no grupo etário mais jovem, 14 a 29 anos de idade, as maiores prevalências para o hábito de beber e suas consequências. No que tange à frequência de consumo, 50,5\% dos jovens bebem com alguma frequência, ao passo que esse comportamento é observado em $44,4 \%$ dos adultos, 30 a 59 anos, e em apenas $21,1 \%$ dos idosos. As prevalências mais elevadas na faixa etária jovem possuem também significância estatística para número de doses em dia típico, frequência de seis ou mais doses, sentir-se culpado após beber e sugestão de outrem para que pare de beber. A faixa etária dos idosos apresenta os maiores índices de abstêmios em todos os itens.

Na Tabela 3 são apresentados os resultados da distribuição entre perfil de consumo e escolaridade. Nessa tabela mostraram-se significantes apenas os itens: frequência de consumo e incapacidade de lembrar o que aconteceu devido à bebida. Quanto à frequência de consumo, o grupo de pessoas com nove ou mais anos de estudo apresenta maiores prevalências de consumo comparado ao grupo com até oito anos de estudo. No grupo de maior escolaridade, $50 \%$ declaram-se abstêmios e $50 \%$ fazem algum tipo de consumo, sendo que $28,7 \%$ consomem pelo menos duas vezes ao mês. O grupo de menor escolaridade apresenta uma prevalência de 37,9\% de indivíduos que fazem algum tipo de consumo, sendo que $23 \%$ consomem duas ou mais vezes ao mês. No que se refere à incapacidade de se lembrar de acontecimentos, provocada pela bebida, o grupo de menor escolaridade apresentou prevalência de 5,4\% de indivíduos que relatam já ter ocorrido, ao passo que no grupo de maior escolaridade esta prevalência é de 10,6\%.

A distribuição do perfil de consumo segundo a renda familiar (Tabela 4) mostrou-se significante apenas para o item frequência de consumo. $\mathrm{O}$ grupo de menor renda apresentou uma prevalência de 58,4\% de abstêmios, enquanto o grupo de maior renda apenas 35\% declararam não beber. Entre os mais abastados, $55 \%$ bebem pelo menos duas vezes por mês, ao passo que entre o grupo de menor renda, 25,2\% bebem com essa mesma frequência. Embora tenha sido observada grande discrepância entre os grupos econômicos da amostra nos outros itens do perfil de consumo, esses resultados não mostraram associação relevante do ponto de vista estatístico.

A análise da Tabela 5, na qual se verificam as respostas de cada item do perfil de consumo distribuídas de acordo com a categoria de pontuação obtida no AUDIT, revela que há significância estatística relevante $(\mathrm{p}<0,001)$ com todos os itens. Observaram-se, conforme esperado, prevalências elevadas de abstinência no grupo AUDIT < 8, ao passo que os perfis de consumo e consequências de risco foram maiores no grupo AUDIT $\geq 8$. Com relação à frequência de consumo, o grupo de risco apresenta prevalência de $86 \%$ para o consumo de bebidas alcoólicas duas ou mais vezes por mês. No que se refere à quantidade de doses, $86 \%$ consomem quatro ou mais doses num dia típico e 38\% consomem seis ou mais doses, em uma ocasião, pelo menos uma vez por semana. Destaca-se ainda que neste grupo, $42 \%$ relataram já ter, devido à bebida, causado algum prejuízo a si mesmo ou a outrem alguma vez na vida. Chama a atenção que no grupo de menor risco, 11,9\% relatam já ter consumido seis ou mais doses em uma ocasião, e $16,4 \%$ afirmam já ter sido interpelado sobre parar de beber.

\section{Discussão}

A identificação do perfil de consumo de bebidas alcoólicas pode ser bastante útil para o entendimento da distribuição de vários aspectos ligados a este hábito. Uma melhor compreensão acerca das características sociodemográficas ligadas a grupos com maior exposição a fatores e comportamentos de risco contribui para a elaboração de políticas e programas de saúde mais eficazes e equânimes.

No presente estudo, a prevalência de pessoas que declararam consumir bebidas alcoólicas com alguma frequência no último ano foi de $43,3 \%$. Esse valor apresenta-se como levemente inferior ao encontrado em levantamento nacional realizado em 108 grandes cidades brasileiras 11 , no qual foi identificado que $49,8 \%$ da população fizeram uso de álcool nos últimos 12 meses. O mesmo estudo identificou que $74,6 \%$ das pessoas no Brasil já fizeram uso de álcool na vida, 38,3\% haviam feito uso nos trinta dias anteriores à entrevista e 12,3\% foram classificados como dependentes. Para o Nordeste, apurou-se que 66,8\% dos entrevistados já fizeram uso de álcool na vida e 13,8\% foram classificados como dependentes. 
Tabela 2

Distribuição do perfil do consumo de bebidas alcoólicas (em \%) segundo faixas etárias. Município de Jequié, Bahia, Brasil, 2010.

\begin{tabular}{|c|c|c|c|c|}
\hline \multirow[t]{3}{*}{ Variável } & \multicolumn{3}{|c|}{ Faixas etárias (anos) } & \multirow[t]{3}{*}{ Valor de $p$ * } \\
\hline & 14-29 & $30-59$ & 60 ou mais & \\
\hline & $(n=97)$ & $(n=135)$ & $(n=38)$ & \\
\hline Frequência de consumo & & & & 0,037 \\
\hline Nunca & 49,5 & 55,6 & 78,9 & \\
\hline Mensalmente ou menos & 20,6 & 17,0 & 13,2 & \\
\hline 2-4 vezes/mês & 26,8 & 23,7 & 2,6 & \\
\hline 2-3 vezes/semana & 2,1 & 3,7 & 2,6 & \\
\hline 4 ou mais vezes/semana & 1,0 & - & 2,6 & \\
\hline Número de doses em dia típico & & & & 0,010 \\
\hline $0-1$ & 53,6 & 65,2 & 92,1 & \\
\hline $2-3$ & 18,6 & 12,6 & 2,6 & \\
\hline $4-5$ & 14,4 & 8,1 & 2,6 & \\
\hline $6-7$ & 4,1 & 5,2 & 2,6 & \\
\hline 8 ou mais & 9,3 & 8,9 & - & \\
\hline Frequência de seis ou mais doses em uma ocasião & & & & 0,005 \\
\hline Nunca & 66,0 & 72,6 & 94,7 & \\
\hline Menos que mensalmente & 20,6 & 11,1 & 2,6 & \\
\hline Pelo menos 1 vez ao mês & 13,4 & 16,3 & 2,6 & \\
\hline Frequência de beber sem conseguir parar & & & & 0,438 \\
\hline Nunca & 92,8 & 95,6 & 97,4 & \\
\hline Menos que mensalmente & 6,2 & 3,0 & - & \\
\hline Pelo menos 1 vez ao mês & 1,0 & 1,5 & 2,6 & \\
\hline Deixou de fazer o esperado devido à bebida & & & & 0,725 \\
\hline Nunca & 95,9 & 94,8 & 100,0 & \\
\hline Menos que mensalmente & 3,1 & 3,7 & - & \\
\hline Pelo menos 1 vez ao mês & 1,0 & 1,5 & - & \\
\hline Necessidade de bebida pela manhã & & & & 0,631 \\
\hline Nunca & 93,8 & 94,1 & 97,4 & \\
\hline Menos que mensalmente & 5,2 & 3,7 & - & \\
\hline Pelo menos 1 vez ao mês & 1,0 & 2,2 & 2,6 & \\
\hline Sentiu-se culpado depois de beber & & & & 0,459 \\
\hline Nunca & 83,5 & 87,4 & 94,7 & \\
\hline Menos que mensalmente & 13,4 & 9,6 & 2,6 & \\
\hline Pelo menos 1 vez ao mês & 3,1 & 3,0 & 2,6 & \\
\hline Incapaz de lembrar o que aconteceu devido à bebida & & & & 0,324 \\
\hline Nunca & 90,7 & 91,1 & 100,0 & \\
\hline Menos que mensalmente & 6,2 & 7,4 & - & \\
\hline Pelo menos 1 vez ao mês & 3,1 & 1,5 & - & \\
\hline Causou ferimentos ou prejuízos após ter bebido & & & & 0,368 \\
\hline Não & 88,7 & 83,7 & 86,8 & \\
\hline Sim, mas não nos últimos 12 meses & 6,2 & 11,9 & 13,2 & \\
\hline Sim, nos últimos 12 meses & 5,2 & 4,4 & - & \\
\hline Alguém sugeriu que parasse de beber & & & & 0,003 \\
\hline Não & 71,1 & 74,8 & 76,3 & \\
\hline Sim, mas não nos últimos 12 meses & 7,2 & 16,3 & 21,1 & \\
\hline Sim, nos últimos 12 meses & 21,6 & 8,9 & 2,6 & \\
\hline
\end{tabular}

* Teste $\chi^{2}$. 
Distribuição do perfil do consumo de bebidas alcoólicas (em \%) segundo anos de estudo. Município de Jequié, Bahia, Brasil, 2010.

\begin{tabular}{|c|c|c|c|}
\hline \multirow[t]{2}{*}{ Variável } & \multicolumn{2}{|c|}{ Anos de estudo } & \multirow[t]{2}{*}{ Valor de $p$ * } \\
\hline & $\leq 8(n=148)$ & 9 ou mais $(n=122)$ & \\
\hline Frequência de consumo & & & 0,058 \\
\hline Nunca & 62,1 & 50,0 & \\
\hline Mensalmente ou menos & 14,9 & 21,3 & \\
\hline 2-4 vezes/mês & 21,6 & 22,1 & \\
\hline $2-3$ vezes/semana & 0,7 & 5,7 & \\
\hline 4 ou mais vezes/semana & 0,7 & 0,9 & \\
\hline Número de doses em dia típico & & & 0,714 \\
\hline $0-1$ & 64,9 & 64,8 & \\
\hline $2-3$ & 12,8 & 13,9 & \\
\hline $4-5$ & 11,5 & 7,4 & \\
\hline $6-7$ & 3,4 & 5,7 & \\
\hline 8 ou mais & 7,4 & 8,2 & \\
\hline Frequência de seis ou mais doses em uma ocasião & & & 0,616 \\
\hline Nunca & 75,0 & 71,3 & \\
\hline Menos que mensalmente & 13,5 & 13,1 & \\
\hline Pelo menos 1 vez ao mês & 11,5 & 15,6 & \\
\hline Frequência de beber sem conseguir parar & & & 0,181 \\
\hline Nunca & 93,9 & 95,9 & \\
\hline Menos que mensalmente & 3,4 & 4,1 & \\
\hline Pelo menos 1 vez ao mês & 2,7 & - & \\
\hline Deixou de fazer o esperado devido à bebida & & & 0,722 \\
\hline Nunca & 96,6 & 95,1 & \\
\hline Menos que mensalmente & 2,7 & 3,3 & \\
\hline Pelo menos 1 vez ao mês & 0,7 & 1,6 & \\
\hline Necessidade de bebida pela manhã & & & 0,487 \\
\hline Nunca & 93,2 & 95,9 & \\
\hline Menos que mensalmente & 4,1 & 3,3 & \\
\hline Pelo menos 1 vez ao mês & 2,7 & 0,8 & \\
\hline Sentiu-se culpado depois de beber & & & 0,139 \\
\hline Nunca & 86,5 & 87,7 & \\
\hline Menos que mensalmente & 8,8 & 11,5 & \\
\hline Pelo menos 1 vez ao mês & 4,7 & 0,8 & \\
\hline Incapaz de lembrar o que aconteceu devido à bebida & & & 0,027 \\
\hline Nunca & 94,6 & 89,3 & \\
\hline Menos que mensalmente & 2,7 & 9,8 & \\
\hline Pelo menos 1 vez ao mês & 2,7 & 0,8 & \\
\hline Causou ferimentos ou prejuízos após ter bebido & & & 0,202 \\
\hline Não & 83,8 & 88,5 & \\
\hline Sim, mas não nos últimos 12 meses & 12,8 & 6,6 & \\
\hline Sim, nos últimos 12 meses & 3,4 & 4,9 & \\
\hline Alguém sugeriu que parasse de beber & & & 0,602 \\
\hline Não & 71,6 & 76,2 & \\
\hline Sim, mas não nos últimos 12 meses & 15,5 & 11,5 & \\
\hline Sim, nos últimos 12 meses & 12,8 & 12,3 & \\
\hline
\end{tabular}

* Teste $\chi^{2}$. 
Tabela 4

Distribuição do perfil do consumo de bebidas alcoólicas (em \%) segundo faixas de renda familiar. Município de Jequié, Bahia, Brasil, 2010.

\begin{tabular}{|c|c|c|c|}
\hline \multirow[t]{2}{*}{ Variável } & \multicolumn{2}{|c|}{ Renda familiar (salários) } & \multirow[t]{2}{*}{ Valor de $p$ * } \\
\hline & $\leq 5(n=245)$ & 6 ou mais $(n=20)$ & \\
\hline Frequência de consumo & & & $<0,001$ \\
\hline Nunca & 58,4 & 35,0 & \\
\hline Mensalmente ou menos & 18,4 & 10,0 & \\
\hline 2-4 vezes/mês & 21,2 & 30,0 & \\
\hline 2-3 vezes/semana & 1,2 & 25,0 & \\
\hline 4 ou mais vezes/semana & 0,8 & - & \\
\hline Número de doses em dia típico & & & 0,768 \\
\hline $0-1$ & 65,3 & 60,0 & \\
\hline $2-3$ & 12,7 & 15,0 & \\
\hline $4-5$ & 9,8 & 10,0 & \\
\hline $6-7$ & 4,1 & 10,0 & \\
\hline 8 ou mais & 8,2 & 5,0 & \\
\hline Frequência de seis ou mais doses em uma ocasião & & & 0,407 \\
\hline Nunca & 73,9 & 60,0 & \\
\hline Menos que mensalmente & 13,1 & 20,0 & \\
\hline Pelo menos 1 vez ao mês & 13,1 & 20,0 & \\
\hline Frequência de beber sem conseguir parar & & & 0,813 \\
\hline Nunca & 94,7 & 95,0 & \\
\hline Menos que mensalmente & 3,7 & 5,0 & \\
\hline Pelo menos 1 vez ao mês & 1,6 & - & \\
\hline Deixou de fazer o esperado devido à bebida & & & 0,148 \\
\hline Nunca & 96,3 & 90,0 & \\
\hline Menos que mensalmente & 2,4 & 10,0 & \\
\hline Pelo menos 1 vez ao mês & 1,2 & - & \\
\hline Necessidade de bebida pela manhã & & & 0,523 \\
\hline Nunca & 93,9 & 100,0 & \\
\hline Menos que mensalmente & 4,1 & - & \\
\hline Pelo menos 1 vez ao mês & 2,0 & - & \\
\hline Sentiu-se culpado depois de beber & & & 0,865 \\
\hline Nunca & 86,9 & 85,0 & \\
\hline Menos que mensalmente & 10,2 & 10,0 & \\
\hline Pelo menos 1 vez ao mês & 2,9 & 5,0 & \\
\hline Incapaz de lembrar o que aconteceu devido à bebida & & & 0,182 \\
\hline Nunca & 92,7 & 85,0 & \\
\hline Menos que mensalmente & 5,3 & 15,0 & \\
\hline Pelo menos 1 vez ao mês & 2,0 & - & \\
\hline Causou ferimentos ou prejuízos após ter bebido & & & 0,394 \\
\hline Não & 86,1 & 80,0 & \\
\hline Sim, mas não nos últimos 12 meses & 10,2 & 10,0 & \\
\hline Sim, nos últimos 12 meses & 3,7 & 10,0 & \\
\hline Alguém sugeriu que parasse de beber & & & 0,478 \\
\hline Não & 73,1 & 80,0 & \\
\hline Sim, mas não nos últimos 12 meses & 14,7 & 5,0 & \\
\hline Sim, nos últimos 12 meses & 12,2 & 15,0 & \\
\hline
\end{tabular}

* Teste $\chi^{2}$. 
Distribuição do perfil de consumo de bebidas alcoólicas (em \%) segundo o escore obtido no Alcohol Use Disorders Identification Test (AUDIT). Município de Jequié, Bahia, Brasil, 2010.

\begin{tabular}{|c|c|c|c|}
\hline \multirow[t]{2}{*}{ Variável } & \multicolumn{2}{|c|}{ Padrão de consumo } & \multirow[t]{2}{*}{ Valor de $p$ * } \\
\hline & AUDIT $<8(n=220)$ & AUDIT $\geq 8(n=50)$ & \\
\hline Frequência de consumo & & & $<0,001$ \\
\hline Nunca & 69,1 & 2,0 & \\
\hline Mensalmente ou menos & 19,1 & 12,0 & \\
\hline $2-4$ vezes/mês & 10,5 & 72,0 & \\
\hline $2-3$ vezes/semana & 1,4 & 10,0 & \\
\hline 4 ou mais vezes/semana & - & 4,0 & \\
\hline Número de doses em dia típico & & & $<0,001$ \\
\hline $0-1$ & 79,1 & 2,0 & \\
\hline $2-3$ & 13,6 & 12,0 & \\
\hline $4-5$ & 5,5 & 28,0 & \\
\hline $6-7$ & 0,5 & 22,0 & \\
\hline 8 ou mais & 1,4 & 36,0 & \\
\hline Frequência de seis ou mais doses em uma ocasião & & & $<0,001$ \\
\hline Nunca & 88,2 & 8,0 & \\
\hline Menos que mensalmente & 10,0 & 28,0 & \\
\hline Mensalmente & 1,4 & 26,0 & \\
\hline Semanalmente & 0,5 & 36,0 & \\
\hline Todos ou quase todos os dias & - & 2,0 & \\
\hline Frequência de beber sem conseguir parar & & & $<0,001$ \\
\hline Nunca & 99,1 & 76,0 & \\
\hline Menos que mensalmente & 0,9 & 16,0 & \\
\hline Mensalmente & - & 4,0 & \\
\hline Semanalmente & - & 2,0 & \\
\hline Todos ou quase todos os dias & - & 2,0 & \\
\hline Deixou de fazer o esperado devido à bebida & & & $<0,001$ \\
\hline Nunca & 99,1 & 82,0 & \\
\hline Menos que mensalmente & 0,9 & 12,0 & \\
\hline Mensalmente & - & 2,0 & \\
\hline Semanalmente & - & 4,0 & \\
\hline Todos ou quase todos os dias & - & - & \\
\hline Necessidade de bebida pela manhã & & & $<0,001$ \\
\hline Nunca & 98,6 & 76,0 & \\
\hline Menos que mensalmente & 1,4 & 14,0 & \\
\hline Mensalmente & - & 6,0 & \\
\hline Semanalmente & - & 2,0 & \\
\hline Todos ou quase todos os dias & - & 2,0 & \\
\hline Sentiu-se culpado depois de beber & & & $<0,001$ \\
\hline Nunca & 95,5 & 50,0 & \\
\hline Menos que mensalmente & 4,5 & 34,0 & \\
\hline Mensalmente & - & 8,0 & \\
\hline Semanalmente & - & 4,0 & \\
\hline Todos ou quase todos os dias & - & 4,0 & \\
\hline
\end{tabular}

(continua) 
Tabela 5 (continuação)

\begin{tabular}{|c|c|c|c|}
\hline \multirow[t]{2}{*}{ Variável } & \multicolumn{2}{|c|}{ Padrão de consumo } & \multirow[t]{2}{*}{ Valor de $p$ * } \\
\hline & AUDIT $<8(n=220)$ & AUDIT $\geq 8(n=50)$ & \\
\hline Incapaz de lembrar o que aconteceu devido à bebida & & & $<0,001$ \\
\hline Nunca & 98,6 & 64,0 & \\
\hline Menos que mensalmente & 0,9 & 28,0 & \\
\hline Mensalmente & 0,5 & 6,0 & \\
\hline Semanalmente & - & 2,0 & \\
\hline Todos ou quase todos os dias & - & - & \\
\hline Causou ferimentos ou prejuízos após ter bebido & & & $<0,001$ \\
\hline Não & 92,3 & 58,0 & \\
\hline Sim, mas não nos últimos 12 meses & 7,3 & 22,0 & \\
\hline Sim, nos últimos 12 meses & 0,5 & 20,0 & \\
\hline Alguém sugeriu que parasse de beber & & & $<0,001$ \\
\hline Não & 83,6 & 30,0 & \\
\hline Sim, mas não nos últimos 12 meses & 13,2 & 16,0 & \\
\hline Sim, nos últimos 12 meses & 3,2 & 54,0 & \\
\hline
\end{tabular}

* Teste $\chi^{2}$.

Índice elevado de abstinência constitui-se em um bom indicador de saúde. No entanto, essa situação não pode ser descuidada e a vigilância constante deste fator deve ser objeto de ação perene de políticas intersetoriais e de saúde 15. No Brasil, a publicidade de bebidas alcoólicas se destaca pela sua qualidade e criatividade 20,21 . Assim, o marketing mercadológico é direcionado a essa população-alvo vista como importante nicho de mercado pela indústria de bebidas alcoólicas.

No que se refere ao número de doses em dia típico, 21,8\% dos entrevistados referiram consumir quatro ou mais doses. Este resultado guarda similaridade com estudo realizado em Campinas (São Paulo) 16, no qual 21,2\% das pessoas referiram consumir mais de três doses tipicamente ao beber. Em estudo realizado no Sul do Brasil 22 observou-se que $14,9 \%$ dos sujeitos pesquisados consumiram quatro ou mais doses numa única ocasião. Por outro lado outros estudos encontraram prevalências superiores de elevado consumo em dia típico. Laranjeira et al. 23 encontraram que, entre os bebedores, $29 \%$ consumiam usualmente cinco ou mais doses.

Importante também analisar a frequência com que o elevado consumo acontece. Os resultados demonstram que $13,3 \%$ das pessoas referiram pelo menos uma vez ao mês consumir seis ou mais doses em uma ocasião. Quantitativo superior ao encontrado em Campinas $16 \mathrm{em}$ que $9,6 \%$ da amostra referiram consumir seis ou mais doses em uma ocasião pelo menos uma vez ao mês.
O padrão de consumo de quatro ou mais doses em uma ocasião é internacionalmente denominado binge drinking 24 , equivalente no Brasil a beber pesado episódico. É especialmente relevante identificar o binge drinking na população, uma vez que está fartamente documentado que este comportamento está intimamente relacionado com diversos problemas de saúde e com casos de violência 5,25 .

A detecção deste tipo de nuance nos padrões de consumo de bebidas alcoólicas de uma população deve servir de alerta para as diversas consequências sociais decorrentes do consumo elevado episódico do álcool. No que se refere a prejuízos por conta de bebida, em Rio Grande (Rio Grande do Sul) 26, 5,4\% responderam que já causaram, mas não no último ano, e 2,4\% responderam que causaram prejuízos, por causa da bebida, no último ano. Estas proporções foram mais elevadas em Jequié, encontrando-se, respectivamente, $10 \%$ e $4,1 \%$. Os prejuízos causados pelo elevado consumo de bebidas alcoólicas, comumente, associam-se aos índices de acidentes de trânsito, brigas, violência contra a mulher, absenteísmo, acidentes de trabalho e até mesmo homicídios 5 . Essa situação pode ser um indicativo de que as políticas para redução da violência, grave problema social contemporâneo, devem perpassar pelo incentivo à diminuição do consumo de álcool.

Um indicativo importante dos problemas causados pelo consumo de bebidas alcoólicas corresponde à proporção de pessoas que admitem já terem sido aconselhadas por um amigo, 
parente e ou profissional de saúde a parar de beber. Essa situação demonstra que o consumo de álcool, rotineiro ou episódico, tem despertado preocupação de outrem sobre esse hábito. Mesmo que o consumidor não perceba, o seu consumo de bebidas alcoólicas tem suscitado inquietação sobre a possibilidade de esse costume fazer mal a si ou a terceiros. Os resultados do estudo demonstraram que $26,3 \%$ da amostra já foram aconselhados a parar de beber, sendo que $12,6 \%$ admitiram terem sido aconselhados nos últimos 12 meses.

Se forem consideradas as pessoas que apresentaram escore $<8$ para o AUDIT, percebe-se que $16,4 \%$ relatam que algum amigo, parente ou profissional de saúde já sugeriu que parasse de beber. Isto mostra que apesar destes indivíduos terem sido classificados como consumidores de baixo risco, as pessoas em seu entorno conseguem perceber sinais de uso nocivo ou prejudicial de bebida alcoólica.

Essas proporções são consideravelmente mais elevadas quando comparadas às encontradas em outros estudos que também utilizaram o AUDIT. Barros et al. 16 encontraram 6,3\% de pessoas que, alguma vez na vida, foram abordados com sugestões para parar de beber. Em Rio Grande 26 , esse percentual foi de $16 \%$, destaquese que $8,1 \%$ relataram que foram aconselhados nos últimos 12 meses.

A ingestão de álcool e o seu consumo de alto risco mantêm forte associação com as relações de gênero. Estudos epidemiológicos 1,20 demonstram que os homens, quando comparados com as mulheres, consomem bebidas alcoólicas em maior frequência, em quantidades mais elevadas e têm se envolvido em episódios de violência ou conduta socialmente reprovada por conta do consumo de álcool. A mortalidade precoce seja em decorrência da violência urbana ou dos acidentes de trânsito, e sua associação com o consumo de bebidas alcoólicas é um problema que atinge os homens em proporção, consideravelmente, mais elevada.

Os resultados encontrados nessa pesquisa corroboram os achados da literatura de maior prevalência de consumo de álcool entre os homens. No entanto, embora menor do que entre os homens, a prevalência de mulheres que consomem álcool com alguma frequência é consideravelmente elevada. Essa situação de elevado consumo de álcool entre as mulheres foi também encontrada em outros estudos 16,22 e apresenta tendência crescente no Brasil 1 . O crescente consumo de bebidas alcoólicas entre as mulheres pode estar associado ao aumento da independência feminina, maior participação no mercado de trabalho e, consequente, ampliação da renda própria. A condição de independência moral e financeira tende a induzir o desenvolvimento de hábitos antes predominante entre os homens, como frequentar bares com amigas para o consumo bebidas alcoólicas.

Outro contexto importante é a distribuição e o perfil do consumo entre as faixas etárias. Os achados concordantes entre este estudo e diversos estudos epidemiológicos no Brasil 11,15,27 mostram que os padrões risco para o consumo de bebidas alcoólicas são mais frequentes nas faixas etárias que poderiam ser classificadas como jovens. Nesta faixa etária, mais da metade das pessoas relatam consumir bebidas alcoólicas, e o padrão de beber em binge é maior quando comparado com os outros dois grupos etários. O hábito de beber em binge entre os mais jovens pode estar associado às descobertas e prazeres da adolescência e a sensação de independência e amadurecimento que o consumo de álcool pode trazer. É comum adolescentes e adultos jovens saírem em grupo para episódios de bebedeiras e não raro é a competição entre eles de quem consegue beber em maior quantidade.

Este padrão de consumo elevado de bebidas alcoólicas entre os jovens tem sido também atribuído ao forte apelo do marketing da indústria de bebidas e do pequeno poder de regulação do estado brasileiro 28. Barbor et al. 2 têm chamado a atenção para o fato de que o álcool não é uma mercadoria comum, e portanto, deve ser alvo de políticas públicas especialmente planejadas, que levem em consideração todos os seus efeitos negativos em nível individual e social, bem como considerando as evidências de custo-efetividade para a sua aplicação.

No presente estudo, dos itens que compõem o perfil de consumo apenas a frequência do consumo apresentou significância com os níveis de renda e escolaridade. O grupo de maior escolaridade e maior renda demonstrou consumo de bebidas alcoólicas de maneira mais frequente. As variáveis socioeconômicas, a exemplo de escolaridade e renda, têm se apresentado em associação controversa com o uso e abuso de álcool. A prevalência de uso e abuso de álcool apresentase em alguns estudos associada a segmentos de maior escolaridade 14,22,27, como também, em outros estudos, apresenta-se associada a menor escolaridade ${ }^{29}$. No que se refere à renda, também há discrepância nos resultados de diversas pesquisas, sendo encontrada tanto associação positiva entre consumo de álcool e renda mais elevada 14,16, como também associação inversa entre essas variáveis 29 . Vargas et al. 27, ao refletirem sobre essa situação, afirmam que a maioria dos estudos encontrou um consumo mais frequente entre os segmentos de maior nível socio- 
econômico. No entanto, é nos segmentos mais pauperizados que o consumo abusivo e de alto risco se faz presente em maior proporção.

É provável que outros fatores culturais e religiosos se conjuguem com as variáveis escolaridade e renda, influenciando de forma complexa o perfil de consumo de bebidas alcoólicas na população. A análise da influência da escolaridade e da renda sobre comportamentos é deveras complicada, sobretudo em países em desenvolvimento, uma vez que incrementos individuais de anos de escolaridade têm um efeito restrito no aumento da renda.

No que se refere ao padrão de consumo, em Jequié $18,5 \%$ da amostra enquadram-se no padrão de risco elevado, pontuação maior ou igual a 8 no AUDIT. Esse resultado é maior do que os encontrados em Campinas 16, 8,4,\%, e Pelotas (Rio Grande do Sul) 29, 14,3\%. Por outro lado, apresenta-se menor do que os resultados de Bebedouro (São Paulo) 27, 21,6 \%. Interessante notar que o consumo de maior risco apresentase em percentuais mais elevados em cidades de menor porte, Jequié e Bebedouro, quando comparado com cidades maiores. Essa situação pode ser um indicativo de que o consumo de álcool apresenta-se característica de maior risco nas cidades de menor porte. Isto pode estar relacionado às dificuldades das cidades menores em desenvolver políticas públicas de cultura, bem como da escassez de equipamentos públicos de lazer e esporte. Como opção de divertimento, as populações desses municípios se inclinariam ao consumo mais elevado de bebidas alcoólicas.

Destaca-se que no grupo de menor risco (AUDIT < 8), foram encontrados indícios de padrões de consumo pesado episódico, o binge drinking, em parcela significativa das pessoas. Estudos 2,15 têm indicado que o beber pesado episódico está associado a mais e maiores problemas físicos, sociais e mentais do que padrões de consumo que se aproximam da dependência.

A investigação epidemiológica do perfil de consumo de bebidas alcoólicas na população maior de 14 anos da zona urbana de Jequié revelou valores que podem ser comparados a outros cenários distintos. Entretanto, todas as comparações devem ser relativizadas por conta de diferenças metodológicas, objetivos, e principalmente pelas características socioculturais diferenciadas das populações em questão. Fatores socioeconômicos e traços culturais acabam por influenciar sobremaneira questões de hábito, tal qual é o consumo de bebidas alcoólicas.

Há que se levar em consideração algumas limitações do estudo a exemplo das dificuldades inerentes a um inquérito domiciliar. Por se tratar de pesquisa que investiga hábitos e comportamentos relacionados à saúde, existe a possibilidade de interferência na fidedignidade das respostas obtidas. A este respeito, é razoável afirmar que os inquéritos domiciliares contendo questões acerca do consumo de bebidas alcoólicas estão sujeitos a diversos vieses, sobretudo os vieses de memória e de resposta, que podem subestimar a frequência e o volume do consumo ${ }^{29}$. No que se refere à amostra, a mesma foi representativa da população em questão para o estudo do perfil de consumo, no entanto, mostrou-se inapropriada para a detecção de desfechos menos comuns.

$O$ estudo foi sensível às diferenças encontradas na distribuição do perfil de consumo de bebidas alcoólicas de acordo com as variáveis sexo, idade, escolaridade e renda. A partir dos resultados obtidos foi possível concluir que a distribuição característica das variáveis estudadas nesta população é compatível com maior frequência e quantidade de uso de álcool entre os homens, quando comparados com as mulheres, e entre os jovens quando comparados com os adultos e os idosos. No que tange à escolaridade e à renda observou-se uma distribuição mais complexa, na qual o consumo revelou-se mais elevado nos grupos de maior escolaridade, bem como nos de maior renda, no entanto, o relato de problemas relacionados ao consumo aparece mais nos grupos de menor escolaridade. A ocorrência do padrão de consumo pesado episódico de álcool foi algo marcante e deve servir de alerta para o fato de que os problemas relacionados ao consumo de bebidas alcoólicas transcendem o problema do alcoolismo.

O entendimento das relações que se conjugam no perfil de consumo de bebidas alcoólicas para a determinação de problemas é multifacetado. As propostas de ação para o seu enfrentamento são igualmente complexas. Neste sentido, para a população estudada são apresentados grupos vulneráveis, ainda não considerados dependentes de bebidas alcoólicas, e que também devem ser alvo de políticas públicas de vigilância, controle de riscos e danos e promoção da saúde. 


\section{Resumo}

Estudo transversal que objetivou verificar o perfil do consumo de bebidas alcoólicas de acordo com sexo, idade, renda e escolaridade na população urbana de Jequié, Bahia, Brasil. Amostra aleatória composta por 270 indivíduos maiores de 14 anos residentes na zona urbana, pesquisados entre janeiro e março de 2010. Como instrumento utilizou-se o Alcohol Use Disorders Identification Test (AUDIT). Os resultados mostraram que 21,8\% consomem quatro ou mais doses em dia típico; 14,1\% admitiram já terem causado algum prejuízo após ter bebido; 26,3\% relataram que alguém já sugeriu que parasse de beber; entre os homens $40 \%$ bebem mais que quatro doses no dia típico; 50,5\% dos jovens bebem com alguma frequência. A distribuição característica das variáveis estudadas é compativel com maior frequência e quantidade de uso de álcool entre os homens e entre os jovens. A ocorrência do padrão de consumo pesado episódico de álcool foi marcante. São apresentados grupos vulneráveis, ainda não considerados dependentes e que também devem ser alvo de políticas públicas de promoção da saúde e prevenção.

Transtornos Relacionados ao Uso de Álcool; Consumo de Bebidas Alcoólicas; Estudos Transversais

\section{Colaboradores}

L. N. Ferreira participou de todas as etapas de desenvolvimento do estudo e elaboração do artigo. Z. N. Sales e C. A. Casotti contribuíram na concepção do estudo e revisão da versão final do artigo. J. P. Bispo Júnior colaborou na análise dos dados, redação do artigo e revisão da versão final. A. C. R. Braga Júnior participou da análise dos dados e da revisão da versão final do artigo.

\section{Agradecimentos}

À Universidade Estadual do Sudoeste da Bahia pelo financiamento da pesquisa (edital 025/2009).

\section{Referências}

1. Galduróz JCF, Carlini EA. Use of alcohol among the inhabitants of the 107 largest cities in Brazil - 2001. Braz J Med Biol Res 2007; 40:367-75.

2. Barbor TF, Caetano R, Casswell S, Edwards G, Giesbrect N, Graham K, et al. Alcohol: no ordinary commodity: research and public policy. $2^{\text {nd }}$ Ed. New York: Oxford University Press; 2010.

3. Burger M, Brönstrup A, Pietrzik K. Derivation of tolerable upper alcohol intake levels in Germany: a systematic review of risks and benefits of moderate alcohol consumption. Prev Med 2004; 39: 111-27.

4. Bye EK, Rossow I. The impact of drinking pattern on alcohol-related violence among adolescents: an international comparative analysis. Drug Alcohol Rev 2010; 29:131-7.

5. Zaleski M, Pinsky I, Laranjeira R, Ramisetty-Mikler $S$, Caetano R. Violência entre parceiros íntimos e consumo de álcool. Rev Saúde Pública 2010; 44:53-9.
6. Silva RA, Jansen K, Godoy RV, Souza LDM, Horta BL, Pinheiro RT. Prevalência e fatores associados a porte de arma e envolvimento em agressão física entre adolescentes de 15 a 18 anos: estudo de base populacional. Cad Saúde Pública 2009; 25: 2737-45.

7. O'Keefe JH, Bybee KA, Lavie CJ. Alcohol and cardiovascular health: the razor-sharp double-edged sword. J Am Coll Cardiol 2007; 50:1009-14.

8. Organização Mundial da Saúde. Classificação estatística internacional de doenças e problemas relacionados à saúde, 10a revisão. v. 1. São Paulo: Centro Colaborador da OMS para a Classificação de Doenças em Português; 1995.

9. Foxcroft DR, Ireland D, Lister-Sharp DJ, Lowe G, Breen R. Longer-term primary prevention for alcohol misuse in young people: a systematic review. Addiction 2003; 98:397-411. 
10. World Health Organization. Global status report on alcohol. Geneva: World Health Organization; 2004.

11. Carlini EA, Galduróz JCF, Noto AR, Nappo SA. II levantamento domiciliar sobre o uso de drogas psicotrópicas no Brasil: estudo envolvendo as 108 maiores cidades do país, 2005. São Paulo: Centro Brasileiro de Informações sobre Dorgas Psicotópicas/Departamento de Psicobiologia, Universidade Federal de São Paulo; 2007.

12. Kerr-Corrêa F, Tucci AM, Hegedus AM, Trinca LA, Oliveira JB, Floripes TMF, et al. Drinking patterns between men and women in two distinct Brazilian communities. Rev Bras Psiquiatr 2008; 30:235-42.

13. Vieira DL, Ribeiro M, Laranjeira R. Evidence of association between early alcohol use and risk of later problems. Rev Bras Psiquiatr 2007; 29:222-7.

14. Almeida-Filho N, Lessa I, Magalhães L, Araújo MJ, Aquino E, Kawachi I, et al. Alcohol drinking patterns by gender, ethnicity, and social class in Bahia, Brazil. Rev Saúde Pública 2004; 38:45-54.

15. Laranjeira RR, Pinsky I, Zlesky M, Caetano R. I levantamento nacional sobre os padrões de consumo de álcool na população brasileira. Brasília: Secretaria Nacional Antidrogas; 2007.

16. Barros MBA, Botega NJ, Dalgalarrondo P, MarínLeón L, Oliveira HB. Perfil do consumo de bebidas alcoólicas: diferenças sociais e demográficas no Município de Campinas, Estado de São Paulo, Brasil, 2003. Epidemiol Serv Saúde 2008; 17:259-69.

17. Barbor TF, Higgins-Biddle JC, Sunders JB, Monteiro MG. AUDIT - the Alcohol Use Disorders Identification Test: guidelines for use in primary care. $2^{\text {nd }} \mathrm{Ed}$. Geneva: World Health Organization; 2001.

18. Castells MA, Furlanetto LM. Validity of the CAGE questionnaire for screening alcohol-dependent inpatients on hospital wards. Rev Bras Psiquiatr 2005; 27:54-7.

19. Lima CT, Freire ACC, Silva APB, Teixeira RM, Farrell $M$, Prince $M$. Concurrent and construct validity of the AUDIT in an urban Brazilian sample. Alcohol Alcohol 2005; 40:584-9.
20. Pinsky I, Jundi SE, Sanches M, Zaleski M, Laranjeira R, Caetano R. Exposure of adolescents and young adults to alcohol advertising in Brazil. Journal of Public Affairs 2010; 10:50-8.

21. Galduróz JCF, Sanchez ZM, Opaleye ES, Noto AR, Fonseca AM, Gomes PLS, et al. Fatores associados ao uso pesado de álcool entre estudantes das capitais brasileiras. Rev Saúde Pública 2010; 44:267-73.

22. Bortoluzzi MC, Traebert J, Loguercio A, Kehrig RT. Prevalência e perfil dos usuários de álcool de população adulta em cidade do sul do Brasil. Ciênc Saúde Coletiva 2010; 15:679-85.

23. Laranjeira R, Pinsky I, Sanches M, Zaleski M, Caetano R. Alcohol use patterns among Brazilian adults. Rev Bras Psiquiatr 2010; 32:231-41.

24. Burazeri G, Kark JD. Prevalence and determinants of binge drinking in middle age in a transitional post-communist country: a population-based study in Tirana, Albania. Alcohol Alcohol 2010; 45:180-7.

25. Brewer RD, Swahn MH. Binge drinking and violence. JAMA 2005; 294:616-8.

26. Mendoza-Sassi RA, Béria JU. Prevalence of alcohol use disorders and associated factors: a populationbased study using AUDIT in southern Brazil. Addiction 2003; 98:799-804.

27. Vargas D, Oliveira MAF, Araújo EC. Prevalência de dependência alcoólica em serviços de atenção primária à saúde de Bebedouro, São Paulo, Brasil. Cad Saúde Pública 2009; 25:1711-20.

28. Vendrame A, Pinsky I, Faria R, Silva R. Apreciação de propagandas de cerveja por adolescentes: relações com a exposição prévia às mesmas e o consumo de álcool. Cad Saúde Pública 2009; 25:359-65.

29. Dias-da-Costa JS, Silveira MF, Gazalle FK, Oliveira SS, Hallal PC, Menezes AMB, et al. Consumo abusivo de álcool e fatores associados: estudo de base populacional. Rev Saúde Pública 2004; 38:284-91.

Recebido em 02/Out/2010

Versão final reapresentada em 05/Mai/2011

Aprovado em 27/Mai/2011 